\title{
Prospective associations between dietary iron intake and serum ferritin concentrations with fracture risk in EPIC-Norfolk men and women
}

\author{
H. Finck ${ }^{1}$, A. Hart ${ }^{1}$, M. Lentjes ${ }^{2}$, A. Jennings ${ }^{1}$, R. Luben ${ }^{2}$, K. -T. Khaw ${ }^{2}$ and A. Welch ${ }^{1}$ \\ ${ }^{1}$ Norwich Medical School, University of East Anglia, Norwich, NR4 7TJ and ${ }^{2}$ Department of Public Health and \\ Primary Care, University of Cambridge, Cambridge, CBI 8RN
}

Age-related muscle and bone loss are closely linked and may share common risk factors including diet ${ }^{(1)}$. Iron may be important for osteoporosis and fracture prevention via its cofactor roles in bone collagen synthesis and vitamin D synthesis ${ }^{(2-3)}$. However, to date, no epidemiological studies have explored the potential for iron to reduce long-term fracture risk. The present study aimed to investigate prospective associations between iron intake and serum ferritin with fracture risk in older British men and women.

This case-cohort study was based on a random sub-cohort of 4000 participants and 1502 participants with fractures who were enrolled in the prospective EPIC-Norfolk cohort study of 25,639 men and women aged 39-77 years. An informed consent was obtained from all participants and the study was approved by the Norwich District Health Authority ethics committee. Iron intake from foods was estimated from a 7-day diet diary and serum ferritin concentrations using a fluoroimmunoassay. After excluding those with incomplete data, fracture risk at the hip, spine and wrist was calculated for sex-specific quintiles of iron intake and serum ferritin using Prentice-weighted Cox proportional hazard ratios (HRs). All analyses were adjusted for age, family history of osteoporosis, body mass index, smoking, physical activity, steroid medication, menopausal status and HRT (women only), energy intake, dietary calcium intake, calcium supplements and vitamin D supplements, and were performed using STATA (version 11, STATA Corp, USA).

Fifty eight percent of the study population were women and mean (SD) age was 60 (10) years. Mean iron intake was 13.4 (4.1) and $10.9(3.4) \mathrm{mg} / \mathrm{d}$ for men and women, respectively, and mean serum ferritin was $115.6(85 \cdot 2)$ and $64 \cdot 7(52 \cdot 8) \mathrm{ng} / \mathrm{ml}$, respectively. Iron intake and ferritin correlated significantly in men $(\mathrm{r}=0.16 ; P<0.05)$ but not in women $(\mathrm{r}=-0.02 ; P>0.05)$. After the median followup of 12.6 years, adjusted spine fracture risk was significantly lower in women in Q5 of both iron intake $(P=0.008)$ and serum ferritin $(P=0.018)$ compared to those in Q1. The association across all quintiles was also significant (iron intake: HR $0.85,95 \% \mathrm{CI} 0.73-0.99$, $P$-trend $=0.041$; ferritin: HR $0.78,95 \%$ CI $0.65-0.94, P$-trend $=0.009)$. No such associations were found at the hip and wrist in women, and at any site in men.

\begin{tabular}{|c|c|c|c|c|c|c|c|c|c|c|}
\hline \multirow[b]{2}{*}{ Site } & \multicolumn{5}{|l|}{ Dietary iron intake } & \multicolumn{5}{|c|}{ Serum ferritin concentrations } \\
\hline & $n$ subjects / fractures & Q1 HR & $n$ subjects / fractures & Q5 HR & $95 \% \mathrm{CI}$ & $P$-trend & Q1 HR & Q5 HR & $95 \% \mathrm{CI}$ & $P$-trend \\
\hline \multicolumn{11}{|l|}{ Men } \\
\hline Hip & $1842 / 112$ & 1.00 & 0.92 & $0.45-1.90$ & $0 \cdot 80$ & $1304 / 79$ & 1.00 & 0.91 & $0 \cdot 40-2 \cdot 08$ & 0.64 \\
\hline Spine & $1808 / 78$ & 1.00 & 1.84 & $0 \cdot 77-4.39$ & $0 \cdot 12$ & $1277 / 50$ & 1.00 & $1 \cdot 13$ & $0 \cdot 50-2 \cdot 56$ & $0 \cdot 87$ \\
\hline Wrist & $1806 / 70$ & 1.00 & 0.98 & $0 \cdot 42-2 \cdot 29$ & 0.75 & $1283 / 50$ & 1.00 & 0.56 & $0 \cdot 22-1 \cdot 39$ & $0 \cdot 31$ \\
\hline \multicolumn{11}{|c|}{ Women } \\
\hline Hip & $2525 / 339$ & 1.00 & 1.57 & $0 \cdot 98-2 \cdot 52$ & 0.65 & $1673 / 231$ & 1.00 & $0 \cdot 93$ & $0.57-1.54$ & 0.57 \\
\hline Spine & $2334 / 124$ & 1.00 & $0.41 * *$ & $0.21-0.79$ & 0.04 & $1538 / 82$ & 1.00 & $0.44 *$ & $0.22-0.87$ & $<0.01$ \\
\hline Wrist & $2409 / 218$ & 1.00 & 0.95 & $0.56-1.62$ & 0.48 & $1585 / 144$ & 1.00 & 0.74 & $0.42-1 \cdot 30$ & 0.37 \\
\hline
\end{tabular}

Iron intake in $Q 1$ and $Q 5$ ranged from 3-10 mg/d and $16-42 \mathrm{mg} / \mathrm{d}$ in men, and from 2-8 mg/d and 13-30 mg/d in women, respectively. Serum ferritin concentrations in 01 and Q5 ranged from 9-48 $\mathrm{ng} / \mathrm{ml}$ and $169-447 \mathrm{ng} / \mathrm{ml}$ in men, and from 8-25 $\mathrm{ng} / \mathrm{ml}$ and $95-443 \mathrm{ng} / \mathrm{ml} \mathrm{in}$ women, respectively.

Q5 is significantly different from $Q 1: * P<0 \cdot 05 ; * * P<0 \cdot 01$.

This prospective study found inverse associations between dietary intakes of iron and serum ferritin with spine fracture risk in women only, possibly due to a higher number of fracture events in women. The potential role of iron in bone health should be explored further.

1. Sirola J and Kröger H (2011) J Osteo 2011, Article ID 536735, 14 pages

2. Hutton JJ, Tappel AL and Udenfriend S (1967) Arch Biochem Biophys 118, 231-240.

3. Jones G, Strugnell SA and DeLuca HF (1998) Physiol rev 78, 1193-1231. 